\title{
ÜBersetzen Und RAHMEn. AUfFüHRUnGen IN GLOBALISIERTEN POP(ULÄR)KULTUREN
}

\author{
Gabriele Klein
}

Pop ist überall. Pop ist ein Muster eines urbanen Lebensstils, das als Zeichensystem global zirkuliert. Anders aber als der Popdiskurs in den 1970ern in der Nachfolge der Kulturindustriethesen von Max Horkheimer und Theodor W. Adorno annahm, führt die globale Zirkulation des Zeichensystems des Pop nicht zwangsläufig zur Vereinheitlichung, sondern, folgt man den Thesen kultureller Globalisierung seit den 1990er Jahren, eher zur Produktion lokaler Differenz.

Wie aber erfolgt diese Produktion von lokaler Differenz? Diese Frage habe ich in verschiedenen Popkulturen, am Beispiel Techno (Klein 1999) und HipHop (Klein/Friedrich 2003), und in der Populärkultur am Beispiel von Tango und Salsa (Klein 2009) untersucht. In diesen Forschungsprojekten lag der Fokus nicht so sehr auf Musik, sondern auf den Körper- und Tanzkulturen und hier vor allem auf den szenespezifischen Aufführungen. Die Ausgangsthese war, dass das soziale < in diesen Kulturen aufgeführt wird, Theatralität und Realität also ineinanderwirken. Die theoretische Grundlage lieferte ein praxeologischer Zugang, der, verbunden mit einem performativitätstheoretischen Ansatz, die lokalen Praktiken in den Blick nahm und das $>$ Wie der Erzeugung lokaler Differenz in den performativen Strategien der Beglaubigung untersuchte. Zu den Untersuchungsfeldern zählten dabei z. B. räumliches Environment, Rituale, Mode, Körpersprache, Gestik, die in lokalen theatralen Settings wie Battles, Raves, Milongas in Szene gesetzt oder medial dokumentiert wurden und damit als Praktiken einer gelungenen, d.h. aus performativitätstheoretischer Sicht, beglaubigten Inszenierung von Authentizität anzusehen sind.

Diese lokalen Performances sind aber nicht als »originär« oder »einzigartig « zu verstehen, da es sich, neben der lokalen Besonderheit, auch immer um kulturelle Übersetzungen handelt. Aus dieser Sicht beruht die Erzeugung von lokaler Differenz auf einem Übersetzungsvorgang, der das 
Zeichen- und Symbolsystem von Popkulturen aktualisiert und transformiert und dabei auf die gelungene Inszenierung von Authentizität angewiesen ist.

Ausgehend von der These der Cultural Studies, dass lokale Aneignung sich nur dann vollzieht, wenn das (pop)kulturelle »Angebot« lebensweltlich relevant ist, verfolgt dieser Text die Frage, wie sich popkulturelle Übersetzungsprozesse vollziehen und lokal gerahmt werden. Damit leistet der Text einen praxeologischen Beitrag zu einer Differenzierung dieser These.

\section{Zur Praxeologie kultureller Übersetzung}

Das Konzept der kulturellen Übersetzung entstammt sowohl einem »cultural turn « in der Übersetzungswissenschaft (Stoll 2008: 177-201) als auch der Etablierung der »postcolonial studies « und vor allem der »translation studies« (Bachmann-Medick 2008: 141-159) im Feld der Sozialwissenschaft. Es findet prinzipiell zwei Anknüpfungspunkte in der kulturtheoretischen Debatte. Zum einen in der Multikulturalismus- und Interkulturalitätsdebatte, die kulturellen Formationen eine Einzigartigkeit und Originalität unterstellt (Buden 2008 u. 2009). Es gibt dieser Debatte zufolge zwar keine universelle Kultur, aber eine Pluralität verschiedener Kulturen, denen ebenfalls essentialistische Identitätskonzepte zugrunde liegen, die sich entweder anerkennen oder (mitunter mit Gewalt) ausschließen. Jede Kultur, so dieses Interkulturalitätskonzept, ist essentiell verbunden mit einer ethnischen, geschlechtlichen oder sexuellen Herkunft: Muslimische und christliche, männliche und weibliche, weiße und schwarze Kulturen sind mit einer Identität ausgerüstet, die möglicherweise interkulturell kommunizierbar, aber nicht überbrückbar ist. Dieses Konzept von Interkulturalität ist zudem eng verknüpft mit dem Begriff des Nationalstaates. Es stellt die konzeptionelle Basis einer staatlichen Politik bereit, die im Sinne der Mehrheit »Kultur macht « und ihre vermeintlich originäre Kultur schützt (American HipHop, Brit-Pop, argentinischer Tango, deutscher Techno etc.).

Ein anderes Konzept kultureller Übersetzung findet seinen Ausgangspunkt in der Idee der Dekonstruktion. Diese Denkrichtung, die von den »postcolonial studies« vertreten wird, fragt nicht nach dem Arrangement der Beziehungen zwischen den Kulturen. Vielmehr geht es darum, die Idee einer originären kulturellen Identität selbst zu unterlaufen. Kultur wird hier nicht auf eine vorgegebene Essenz zurückgeführt, sondern als ein System von Zeichen verstanden, die sich aufeinander beziehen, die Spuren hinterlassen und ihre eigenen Ursprünge in sich selbst haben. Deutsch sein, schwarz oder schwul sein ist demnach einzig das Produkt einer kulturellen 
Aktivität, die selbst als ein Prozess der Übersetzung verstanden wird. »In the discipline of semiotics of culture it comes naturally to say that culture is translation, and also that translation is culture (Torop 2002: 603).

Der Ausgangspunkt für diese dekonstruktivistische Kulturtheorie der Übersetzung, an die sich meine Argumentation anlehnt, liegt in dem Aufsatz Walter Benjamins »Die Aufgabe des Übersetzers« (Benjamin 1972) begründet. Hier übt Benjamin Kritik an der binären Übersetzungstheorie, die Friedrich Schleiermacher (1973) Anfang des 19. Jahrhunderts entwickelte und die zum grundlegenden Paradigma der Übersetzungswissenschaft wurde. Schleiermacher geht von zwei binären Möglichkeiten der Übersetzung aus: Originaltreue oder ästhetische Wirkung. Ähnlich wie Wilhelm von Humboldt (Humboldt/Güthling 1926) setzt Schleiermacher auf Originaltreue. Seiner Ansicht nach sollen die Leser das Gefühl haben, »dass sie ausländisches vor sich haben « (Schleiermacher 1973: 54). Der »Geist der Sprache« des Originals soll demnach in der Übersetzung sichtbar werden, die Übersetzung hat sich folglich an der Sprache des Originals auszurichten. Dadurch sei das Fühlen des Fremden und damit letztendlich Sprach- und Kulturvermittlung möglich. Übersetzung, sowohl im Sinne der Verfremdung als auch der Einbürgerung, bildet dabei die Brücke zwischen den Sprachen, Kulturen, Nationen oder Kontinenten.

Benjamin räumt mit der Binarität von Original und Kopie auf, die die Übersetzungswissenschaft bis Anfang des 20. Jahrhunderts prägte. Benjamin verwendet die Metapher der Tangente, die den Kreis (das Original) nur einmal berührt und dann ihren Weg nimmt. Es gibt demnach keine Ausgangsund Zielkultur, die miteinander zu verbinden wären. Übersetzung versteht Benjamin als Wandlung und Erneuerung; sie ist damit selbst Kultur wie Kultur eine permanente Übersetzung ist.

Hier knüpft die dekonstruktivistische Theorie mit dem Konzept der Übersetzung an: Homi Bhabha nennt diesen Raum der Übersetzung den Raum der Hybridität. Es ist jener »Third Space (Rutherford 1990: 207-211; Bhabha 2000), in dem Transformation oder Transgression möglich ist, indem sich binäre kulturelle Codes verändern können und etwas Neues entstehen kann. Allerdings wäre es falsch, diesen Raum des Hybriden als einen besonderen kulturellen Raum anzusehen. Kultur ist Übersetzung, ständig im Übergang. »Was Transkultur genannt wird«, so Gayatri Chakravorty Spivak, »ist Kultur in ihrem Vollzug. Transkulturation ist nichts Besonderes oder Andersartiges, sie ist ein Moment in einer Taxonomie der Normalität dessen, was Kultur genannt wird«(Spivak 2008, o.S.). Transkultur als etwas Spezifisches anzusehen, bedeutet hingegen, Kultur in einen bestimmten politischen (zumeist nationalen) Kontext zu stellen. Hier zeigt sich die Notwendigkeit einer 
politischen Kontextualisierung des Konzeptes der kulturellen Übersetzung, vollzieht sich diese doch immer in einem Machtfeld politischer und symbolischer Ökonomien.

Dieser Lesart zufolge verweist kulturelle Übersetzung weder auf einen Anfangs- und Endpunkt noch auf ein Original. Die Vorstellung von Kultur als Einheit, ausgestattet mit einer zeitlichen Linearität und einer Originalität, entsteht vielmehr erst im Akt der Übersetzung, retrospektiv, wie Barbara Johnson in ihrem Buch »Mother Tongues« (Johnson 2003) ausführt, in dem sie sich mit Benjamins Text befasst und dessen Thesen pointiert. Die Übersetzung selbst ist es demnach, die eine vermeintlich ursprüngliche Einheit als Schein entlarvt. Und genau hier zeigt sich Übersetzung als Machtstrategie. An diese Lesart anknüpfend nennt Tomislaw Longinovic (Longinovic 2002; Longinovic/Buden 2008) kulturelle Übersetzung »die Praxis des Alltagslebens «. Sie ist eine performative Praxis und gerade dadurch ein Muster von Subjektivierung.

Kulturelle Übersetzung stellt immer auch die Macht-Frage. Denn seit dem »cultural turn« hat Kultur nicht, wie so oft angenommen (Goehler 2006), den Begriff der Gesellschaft von der politischen Bühne verdrängt und dessen führende Rolle übernommen. Indem politische Konflikte und Kämpfe kulturalisiert worden sind oder Kulturdialog zum Thema der internationalen politischen Beziehungen erhoben wurde, ist vielmehr Kultur selbst zur politischen Bühne geworden, kulturelle Übersetzung ist somit auch immer eine politische Kategorie, die sowohl subversiv als auch affirmativ wirken kann.

Kulturelle Übersetzung aus dieser Lesart meint nicht Kulturverstehen, meint nicht Brückenbau. Sie fokussiert nicht eine (vermeintliche) Ausgangsund Zielkultur, sondern zielt darauf $a b$, »Zwischenräume « jenseits des binären Bauprinzips zu erschließen und diese als Aushandlungsräume für kulturelle Übersetzungsprozesse zu untersuchen. Ein praxeologischer Zugang zu kultureller Übersetzung stellt die Frage, wie diese komplexen kulturellen Austausch- und Aushandlungsprozesse sich vollziehen und zwar jenseits eines Transfers zwischen Original und Übersetzung: zum einen im Sinne mehrdimensionaler Transformationen in Zwischenräumen, zum anderen mit Blick auf die Praktiken der Übersetzungen.

Zwischen Aneignung und »Seinlassen«, Anverwandlung und Unaufhebbarkeit von Alterität ist damit ein weiter Bogen gespannt, der die Problematik einer »Über-Setzung « zwischen Kulturen betrifft. Zudem verschiebt sich mit einer praxeologischen Perspektive die Thematisierung des transkulturellen Übersetzens sowie postkolonialer Praktiken im Umgang mit anderen Kulturen auf die den kulturellen Übersetzungen zugrunde liegenden alltäglichen und körperlich gebundenen Praktiken. So sind in globalisierten Pop(ulär)kul- 
turen erstens unterschiedliche Übersetzungspraktiken »alltäglich« und werden in divergierenden Ausprägungen und »Ver-Wendungen« gebraucht, die wiederum plurale Effekte und Missverständnisse erzeugen. Zweitens bringen diese Praktiken ihre je eigenen Grenzen und »Unübersetzbarkeiten« erst hervor. Diese praxeologische Neuakzentuierung bedeutet demnach eine Rückführung des generellen Problems der Alterität einer jeden Übersetzungstheorie zu einer Untersuchung des jeweiligen »Übersetzungshandelns «, der Praktiken und ihrer performativen Effekte. Damit wird auch ermöglicht, den Übersetzungsbegriff über seine latente Sprachauszeichnung hinaus auf alle Arten kultureller Transformation auszuweiten (z.B. Text/Bild; Musik; Theater/Performance, Tanz/Film etc.).

Ausgehend von dieser kultur- und medientheoretischen Positionierung habe ich kulturelle und situationale Aushandlungsräume und Praktiken kultureller Übersetzungen untersucht. Die Frage lautet, wie die komplexen Prozesse der Hervorbringung und Aneignung von Pop sich praktisch vollziehen und zwar auch jenseits der Vorstellung einer Zeichenkorrespondenz von Ausgangs- und Zielkultur oder eines Transfers eines (vermeintlichen) Originals, sondern im Sinne mehrdimensionaler, mitunter brüchiger medialer Transformationen. Dabei rückt das mediale »Dazwischen«, das sich nicht mehr als Zwischenraum zwischen $\gg A$ « und $\gg B$ « lokalisieren und nicht mehr auf gegebene Räume, auf Ausgangs- und Endpunkt, zurückführen lässt, in den Mittelpunkt des Forschungsinteresses.

\section{Rahmen und Rahmung (pop-)kultureller Übersetzungen}

Um verstehbar zu werden und lebensweltlich relevant zu sein, müssen kulturelle Übersetzungen gerahmt werden. Mit der These, dass Aneignungsprozesse sich nur dann vollziehen, wenn sie lebensweltlich relevant sind, haben die Cultural Studies bereits vor vielen Jahren ein wichtiges Stichwort markiert. »Aneignung « aber, wenn man diesen Begriff verwenden möchte, da er ja ein klassisches Übersetzungsmodell impliziert, muss also gerahmt, d.h. sinnweltlich gedeutet werden. Wie also kann man dies theoretisch fassen? Wie kann eine Praxeologie kultureller Übersetzungen mit einem Rahmenkonzept in Verbindung gebracht werden?

In der Soziologie hat Erving Goffman das Rahmen-Konzept wesentlich geprägt. Er versteht Rahmen als Organisationsprinzipien menschlicher Erfahrung und Interaktion, die es erlauben, Situationen zu definieren (Goffman 1974). Im Unterschied zu neueren kognitionswissenschaftlichen Ansätzen 
verortet Goffman Rahmen nicht allein im menschlichen Bewusstsein, sondern im Bereich von sozialen Handlungen und kollektiven Aktivitäten, die wiederum selbst erst durch Rahmungen ihren Sinn erhalten. „Primäre Rahmen«, d.h. natürliche oder als natürlich wahrgenommene Ereignisse, die gleichwohl auf kollektiven Schemata beruhen, können ihm zufolge moduliert werden (z.B. wenn Alltagsgesten in tänzerische Gesten im HipHop transferiert werden), wobei diese Modulationen über ein System von Konventionen erfolgen. Die Arten der Modulationen sind begrenzt, weil sie als »Übersetzungen « von einem Rahmen in einen anderen erfolgen - ein Vorgang, den Goffman mit dem Begriff »Key« (dt.: »Modul«; Goffman 1974: 56, Fn 14) bezeichnet. Das Modul zeigt an, wie eine Handlung, eine Szene oder eine Situation zu verstehen ist, ähnlich wie die Notation einer Ballettchoreografie. Aufgrund der Existenz derartiger Modulationen sind primäre Rahmungen fragil und permanent gefährdet. Gleichwohl kommt ihnen die Aufgabe der Organisation von Erfahrung zu, wobei diese als soziale Praxis verstanden wird, der Individuen folgen.

Im deutschsprachigen Raum wird, anknüpfend an Goffmans soziologische Rahmen-Analyse, zumeist das an das bildnerische Artefakt angelehnte Substantiv »Rahmen« verwendet. Aus praxeologischer Perspektive aber rückt das Verhältnis von Rahmen als Substantiv und rahmen als Verb in den Vordergrund: Die Verbform »rahmen« bezeichnet das Prozessuale, den konkreten Vollzug, die Praxis des Rahmens. Diese Praxis verstehe ich zugleich als eine Praxis der Aktualisierung, Re-Formulierung, Transformation oder Neukonstruktion bestehender Rahmungen. Dazu zählen in globalisierten Popkulturen insbesondere mediale Rahmungen, die die Wahrnehmung steuern und Möglichkeiten der Aneignung bereitstellen, zugleich aber auch durch diese Praktiken der Aneignung transzendiert und transformiert werden können. Rahmungen erfüllen demnach immer auch eine epistemologische Funktion, insofern Sinn sich »in« Rahmungen als Bedingungen unterschiedlicher »Als«-Funktionen konstituiert. Indem Rahmen ein- und ausgrenzen, implizieren sie nicht nur Differenzsetzungen, sondern bringen etwas »als« Inklusion oder »als« Exklusion, z. B. »als« Sichtbares oder »als« Unsichtbares usw. hervor. Der »Rahmenwechsel « ist demnach nicht als ein singulärer Akt zu verstehen, sondern als ein fortwährender Vorgang der De-, Re- und Neukontextualisierung, der sich im Wechselspiel von Wahrnehmung und Deutung ereignet und ein bestimmtes Rahmenwissen voraussetzt. Das Gelingen der Übersetzung in Form einer Neukonstitution von Sinn ist wesentlich von dem Verstehen dieser komplexen Rahmungsbedingungen und Rahmungserzeugungen abhängig. Rahmungen werden von den Partizipierenden aktiv, wenngleich nicht unbedingt bewusst (mit) hergestellt, zugleich 
sind verschiedene Akteure, zu denen neben Menschen z. B. auch Dinge, Bedingungen oder technische Apparate gehören können, an ihnen beteiligt (Latour 2007).

Vor dem Hintergrund eines solchen praxeologischen Ansatzes, der die Praktiken der Hervorbringung und Aneignung von Pop untersucht, sind Rahmung und Übersetzung nicht als stabile, fixierte Formate, sondern selbst als transitorische Kontextualisierungen zu verstehen, deren Transformationen durch die miteinander verschränkten Praktiken des Rahmens und Übersetzens selbst bedingt sind.

\section{Popkulturelle Übersetzungen und lokale Rahmungen}

Globalisierte Pop(ulär)kulturen lassen sich als transkulturelle Aushandlungs»räume« verstehen. An ihnen lässt sich das Konzept der kulturellen Übersetzung anschaulich machen. Das, was Bhabha für Kultur allgemein konstatiert, gilt insbesondere für die globalisierten Pop(ulär)kultur. Sie

»ist sowohl transnational als auch translational. [...] Die transnationale Dimension kultureller Transformation - Migration, Diaspora, De-Platzierung, Neuverortung - lässt den Prozess kultureller Translation zu einer komplexen Form der Signifikation werden. Der natürliche oder naturalisierte, einheitsstiftende Diskurs [...] kann hier kaum als Bezugspunkt dienen. Der große, wenngleich beunruhigende Vorteil dieser Situation besteht darin, dass sie uns ein stärkeres Bewusstsein von der Kultur als Konstruktion und von der Tradition als Erfindung verschafft« (Bhabha 2000: 257).

Lokale popkulturelle Szenen lassen sich mit Peter Berger und Thomas Luckmann (1969) als symbolische Sinnwelten beschreiben, die durch bestimmte Locations und deren Environments sowie in den szenespezifischen theatralen Aufführungen wie Battles, Konzerten, Jams, Milongas, Salsatecas über soziale Regeln, symbolische Codes, soziale Interaktionen, ritualisierte Handlungen, tänzerische Praktiken und subjektive Gefühlswelten hergestellt und inkorporiert werden (Klein/Haller 2008: 51-74). Räumlich verortet rahmen die Narrative das individuelle Handeln, Fühlen und Denken der Akteure, wobei diese »Produktion von Lokalität « (Appadurai 1998: 11-40 und 1995: 204-225) nicht als eine bloße Reproduktion oder Nachahmung verstanden werden kann. Vielmehr ist der Performanz der sozialen, rituellen und tänzerischen Praktiken das transformatorische Potential der Übersetzung eingeschrieben, das zum einen zu einer Produktion lokaler Differenz in globalisierten Popkulturen führt. Zum anderen lässt es die Erfahrung des 
Pop, vor allem im Tanz, als einen das globale Narrativ transformierenden, da von den kulturell und sozial geprägten Körpern vollzogenen Vorgang werden. Das globale Narrativ Pop wird über Bilder, Texte und Töne als imaginierte Sozialwelt hervorgebracht, in verschiedene kulturelle Kontexte übersetzt und lokal im Zusammenspiel mit globalen Übersetzungsbewegungen verankert.

Globalisierte Pop(ulär)kulturen beruhen auf einem mehr oder weniger geordneten, aber nicht unbedingt hierarchischen System von Erzählungen. Diese Erzählungen fungieren als Sinnproduzenten; sie sind konstitutiv für die Identität der Akteure aber auch für die kollektive Identität der lokalen Szenen. Der Begriff »Narrativ« meint dabei nicht primär die erzählte Geschichte selbst, sondern die Praxis des Erzählens. Diese verweist auf eine erzählte Geschichte (z.B. die imaginierte Sozialwelt des Tango Argentino oder das Ghetto im HipHop), fügt damit das Erzählen selbst in die lineare Ordnung des Zeitlichen ein und setzt auf diese Weise die Erzählung permanent fort « (Müller-Funk 2008: 29). Es ist demnach nicht die globale Erzählung der jeweiligen Popkultur selbst, sondern es sind die performativen Strategien des Erzählens, die Sinn stiften: Zum einen die mit der NeuKontextualisierung einhergehende fortwährende »Produktion von Lokalität « in stadtbezogenen Szenen, die über das Muster sozialer In- und Exklusion soziale und lokale Identitäten stiften. Das permanente Erzählen von »Geschichten « in Bildern, Texten sowie in sozialen Praktiken von Aufführungen ist ein komplexer, widersprüchlicher, brüchiger, zwischen Globalem und Lokalem wechselnder, translokaler Übersetzungsprozess, der konstitutiv sowohl für die Erfahrung wie für die Bildung von Ich-Identität und kollektiver Identität werden kann. In diesem Prozess der Identifizierung wird zugleich die »Erzählung Pop« aktualisiert, entsprechend des lokalen, kulturellen und biographischen Rahmens transformiert und als transformierte lokale Praxis über die Migration von Akteuren oder über mediale Repräsentationen der jeweiligen Kultur wieder in den globalen Prozess übersetzt.

\section{(Pop-)Kulturelle Identitäten}

Aneignungsprozesse sind identitätsstiftend. Das hier zugrunde gelegte Identitätskonzept lehnt sich an Paul Ricoeur an (Ricoeur 1988 und 1991: 392400). Die personale Identität beispielsweise eines Ravers, HipHoppers oder Tango-Tänzers sowie die kollektiven Identitäten in den lokalen Szenen entwickeln sich demnach aus der Produktion und Rezeption von »fiktiven« Geschichten, wie z.B. die Erzählungen über Gangsta-Rap oder über Tango als 
verruchtem Milieutanz, und »faktischen « Geschichten, wobei das »Fiktive« und das »Faktische « in der Erzählung nicht zu trennen sind. Die Narrative produzieren lebensweltliche Muster und Identitätsentwürfe, die sich beispielsweise im Bild und Selbstverständnis der HipHopper oder der Tanguera und des Tangueros niederschlagen. Sie helfen, kontingente Ereignisse wie die szenespezifischen theatralen Aufführungen (z.B. Konzerte, Battles, Parties, Jams, Milongas) in das globalisierte Erzählen der Geschichte zu integrieren und dies mit der eigenen Lebensgeschichte zu verknüpfen. Die narrative Identität selbst wird dabei zum Garanten für die Wahrheit, Originalität und Authentizität des Imaginierten (Anderson 1988: 20). Der von Ricoeur beschriebene mimetische Kreislauf erstens des Verstricktseins in Erzählungen (im Akt des Tanzens oder Musik-Machens beispielsweise), zweitens des expliziten Erzählens im Sinne des darüber Sprechens (in Face-toFace-Kommunikation, aber auch im Internet in Blogs oder auf Facebook z.B.) (Klein/Haller 2006: 233-248) sowie drittens dessen Aktualisierung durch Interpretation, umschreibt die weite Bedeutung des Begriffs »Narrativ«: Es bezieht sich nicht nur auf sprachliche oder textuelle, sondern auch auf so genannte nicht-sprachliche Medien, d.h. auf Ereignisse, Erlebnisse, auf Feste, auf körperliche Aktivitäten. Auch sie finden ihren Referenzrahmen und ihre Legitimation in Narrativen, werden mit Verweis auf das globale Narrativ Pop z. B. als authentisch erlebt, und wirken durch Interpretation des Erlebens identitätsbildend.

Die Übersetzungsbewegungen popkultureller Narrative veranschaulichen die Verbindungen zwischen der Makroebene der global zirkulierenden Bilder, Codes und Zeichen, die die jeweilige popkulturelle Szene prägen, und der Mikroebene der lokalen (Aneignungs-)Praktiken und Identitätsbildungen. Dabei ist gerade das Reden über das »Nicht-Sprechbare « der (affektiv, sexuell oder erotisch aufgeladenen) Erfahrung und die Thematisierung des Verfehlens ein wesentlicher Teil des Narrativs des Pop. Hier setzt die Idee der Retroperspektive ein: Erst in der und durch die Übersetzung wird in den Erzählstrategien eine bestimmte Praxis des Pop retrospektiv als das Original und eine andere als Verfälschung, als Fake markiert und zugleich über diese binäre Setzung eine bestimmte Praxis als authentisch essentialisiert.

Narrative aktualisieren sich in den globalen Pop(ulär)kulturen über Medien: Die Schrift, das Bild, die Musik, die mündliche Erzählung und das Tanzen als körperliches Medium: Gerade das Tanzen aktualisiert die Narrative wie umgekehrt das Tanzen selbst erst in Bezugnahme auf die Narrative essentialisiert, d.h. als »echt«, »authentisch« oder »natürlich« beglaubigt wird. 
Das globale Narrativ einer jeden Pop(ulär)kultur transformiert kulturelle Disparatheiten, die in den unterschiedlichen nationalen (Pop-)Kulturen (z.B. Deutscher HipHop, Brit-Pop), in lokalen Szenen oder in den Geschlechterkulturen des Pop (z.B. riot grrrls) erzeugt werden in Vorstellungen von einem homogenen Raum des Pop. Dies zeigt sich in den Zeichensystemen der weltweiten HipHop- oder der Tangokultur, die in nahezu allen lokalen Szenen übernommen werden, wie Farben, Kleidung, Accessoires, Rituale, ritualisierte Handlungen und Interaktionsordnungen. Und es demonstriert sich in einer linearen Zeitordnung, also einer Popkulturgeschichte, die Tradition schafft, kanonisiert und kulturelle und soziale Orientierung bietet und zugleich zur Mythenbildung und mit ihr zur Etablierung einer Ausgangskultur beiträgt, die retrospektiv als authentisch essentialisiert wird. Mit anderen Worten: Die kulturellen Repräsentationen, wie sie sich in den lokalen Popkulturen und ihren Aufführungen zeigen, werden in der Praxis, verstanden als ein dreifacher Akt des Erzählens im Ricoeurschen Sinne, aktualisiert, bestätigt, selektiert, transformiert und konventionalisiert.

Ereignisse wie Battles, Konzerte oder Milongas sind Rahmungen, die die Mythen beglaubigen und auf diese Weise die Popkulturen als Orte der Außeralltäglichkeit legitimieren. Genau hier - in dem fragilen Möglichkeitsraum der Translation - liegt zugleich das poetische Potential des Pop: in der Unvorhersehbarkeit und Unplanbarkeit der Übertragungen der kulturellen Narrative. Und diese vollziehen sich in globalisierten Pop(ulär)kulturen, wie ich zeigen wollte, nicht nur in den performativen Akten der Wahrnehmung, Inkorporierung, der körperlichen Ko-Präsenzen und der theatralen Aufführungen, sondern in komplexen kulturellen Übersetzungen und vielfältigen Rahmungen sowie in den Verflechtungen der Praktiken der performativen Erzählstrategien des Übersetzens und Rahmens und ihrer mündlichen und schriftlichen sowie ihrer körperlichen, bildlichen und textuellen Medien. 


\section{Literatur}

Anderson, Benedict R. O'G. (Hg.) (1988). Die Erfindung der Nation. Zur Karriere eines folgenreichen Konzepts. Frankfurt/M.: Campus.

Appadurai, Arjun (1995). »The Production of Locality. «In: Counterworks. Managing the Diversity of Knowledge. Hg. v. Richard Fardon. London: Routledge, S. 204225.

Appadurai, Arjun (1998). »Globale ethnische Räume. Bemerkungen und Fragen zur Entwicklung einer transnationalen Anthropologie.«In: Perspektiven der Weltgesellschaft. Hg. v. Ulrich Beck. Frankfurt/M.: Suhrkamp, S. 11-40.

Bachmann-Medick, Doris (2008). „Übersetzung in der Weltgesellschaft. Impulse eines >translational turn «.« In: Kultur, Übersetzung, Lebenswelten. Beiträge zu aktuellen Paradigmen der Kulturwissenschaften. Hg. v. Andreas Gipper und Susanne Klengel. Würzburg: Königshauen \& Neumann, S. 141-159.

Benjamin, Walter (1972). »Die Aufgabe des Übersetzers. «In: Gesammelte Schriften. Bd. IV/1. Hg. v. Rolf Tiedemann und Hermann Schweppenhäuser. Frankfurt/M.: Suhrkamp, S. 9-21.

Berger, Peter L. / Luckmann, Thomas (Hg.) (1969). Die gesellschaftliche Konstruktion der Wirklichkeit. Eine Theorie der Wissenssoziologie. Frankfurt/M.: Fischer.

Bhabha, Homi K. (Hg.) (2000). Die Verortung der Kultur. Tübingen: Stauffenburg.

Buden, Boris (2008). »Kulturelle Übersetzung. Warum sie wichtig ist, und wo damit anzufangen ist. «In: translate.eipcp.net, http://translate.eipcp.net/transversal 10606/buden/de (Version vom 7.12.2008; Stand vom 12.5.2012).

Buden, Boris (2009). »Kulturelle Übersetzung. «In: IG Kultur Österreich, http:// igkultur.at/igkultur/kulturrisse/1150793894/1150797107 (Version vom 19.3.2009; Stand vom 12.5.2012).

Chakrabarty, Dipesh (Hg.) (2002). Habitations of Modernity. Essays in the Wake of Subaltern Studies. Chicago: University of Chicago Press.

Goehler, Adrienne (Hg.) (2006). Verflüssigungen. Wege und Umwege vom Sozialstaat zur Kulturgesellschaft. Frankfurt/M., New York: Campus-Verlag.

Humboldt, Wilhelm von / Güthling, Otto (Hg.) (1926). Aischylos Agamemnon übersetzt von Wilhelm von Humboldt (1816). Leipzig: Reclam (2. Aufl.).

Johnson, Barbara (Hg.) (2003). Mother Tongues. Sexuality, Trials, Motherhood, Translation. Cambridge, MA, London: Harvard University Press.

Klein, Gabriele (Hg.) (1999). Electronic Vibration. Pop, Kultur, Theorie. Hamburg: Rogner \& Bernhard bei Zweitausendeins.

Klein, Gabriele (Hg.) (2009). Tango in Translation. Tanz zwischen Medien, Kulturen, Kunst und Politik. Bielefeld: transcript.

Klein, Gabriele / Friedrich, Malte (Hg.) (2003). Is this real? Die Kultur des HipHop. Frankfurt/M.: Suhrkamp.

Klein, Gabriele / Haller, Melanie (2006). »Präsenzeffekte. Zum Verhältnis von Bewegung und Sprache am Beispiel lateinamerikanischer Tänze.«In: Body Turn. Perspektiven der Soziologie des Körpers und des Sports. Hg. v. Robert Gugutzer. Bielefeld: transcript, S. 233-248.

Klein, Gabriele / Haller, Melanie (2008). „Café Buenos Aires und Galeria del Latino. Zur Translokalität und Hybridität städtischer Tanzkulturen. «In: Bewegungsraum und Stadtkultur. Sozial- und kulturwissenschaftliche Perspektiven. Hg. v. Jürgen Funke-Wieneke und Gabriele Klein. Bielefeld: transcript, S. 51-74. 
Latour, Bruno (2007). Eine neue Soziologie für eine neue Gesellschaft. Einführung in die Akteur-Netzwerk-Theorie. Frankfurt/M.: Suhrkamp.

Longinovic, Tomislaw (2002). »Fearful Asymmetries. A Manifesto of Cultural Translation. « In: The Journal of the Midwest Modern Language Association 35, H. 2, S. 5-12.

Longinovic, Tomislaw / Buden, Boris (Hg.) (2008). »Die Antwort liegt in der Übersetzung. «In: transversal.eipcp.net, http://eipcp.net/transversal/0908/longino vic-buden/de (Version vom 5.12.2008; Stand vom 12.5.2012).

Müller-Funk, Wolfgang (Hg.) (2008). Die Kultur und ihre Narrative. Wien, New York: Springer.

Ricoeur, Paul (Hg.) (1988). Zeit und Erzählung I. München: Fink.

Ricoeur, Paul (1991). »Die erste Aporie der Zeitlichkeit: die narrative Identität.« In: Zeit und Erzählung III. Hg. v. dems. München: Fink, S. 392-400.

Rutherford, Jonathan (1990). »The Third Space. Interview mit Homi K. Bhabha. «In: Identity. Community, Culture, Difference. Hg. v. dems. London: Lawrence \& Wishart, S. 207-211.

Schleiermacher, Friedrich (1973/1838). „Über die verschiedenen Methoden des Übersetzens. «In: Das Problem des Übersetzens. Hg. v. Hans Joachim Störig. Darmstadt: Wissenschaftliche Buchgesellschaft (2. Aufl.), S. 38-70.

Spivak, Gayatri Chakravorty (2008). „Weitere Überlegungen zur kulturellen Übersetzung. «In: translate.eipcp.net, http://translate.eipcp.net/transversal/0608/ spivak/de (Version vom 6.12.2008; Stand vom 12.5.2012).

Stoll, Karl-Heinz (2008). »Translation als Kreolisierung."In: Kultur, Übersetzung, Lebenswelten. Beiträge zu aktuellen Paradigmen der Kulturwissenschaften. Hg. v. Andreas Gipper und Susanne Klengel. Würzburg: Königshauen \& Neumann, S. $177-201$.

Torop, Peeter (2002). »Translation as Translating as Culture.«In: Sign Systems Studies 30, H. 2, S. 594-605.

\begin{abstract}
This text deals with a praxeological perspective on the translocality of pop cultures. Based on the theory of Cultural Studies that local occupancy does only occur when the (pop)cultural »supply« is lifeworldly relevant, the text discusses the question, how pop-cultural translation processes take place and are framed locally. It investigates from the perspective of performance theory the production strategies of local differences through performative practices of authentification in scene-specific performances. Thereby the text makes a praxeological contribution to a differentiation of the above-mentioned thesis of Cultural Studies.
\end{abstract}

